University of Nebraska - Lincoln

DigitalCommons@University of Nebraska - Lincoln

2011

\title{
Anisotropy of zigzag chains of palladium
}

P. K. Sahota

The LNM Institute of Information Technology Jaipur, Rajasthan, India

Ralph A. Skomski

University of Nebraska-Lincoln, rskomski2@unl.edu

Axel Enders

University of Nebraska-Lincoln, a.enders@me.com

David J. Sellmyer

University of Nebraska-Lincoln, dsellmyer@unl.edu

Arti Kashyap

University of Nebraska-Lincoln, akashyap@Inmiit.ac.in

Follow this and additional works at: https://digitalcommons.unl.edu/physicsenders

Part of the Physics Commons

Sahota, P. K.; Skomski, Ralph A.; Enders, Axel; Sellmyer, David J.; and Kashyap, Arti, "Anisotropy of zigzag chains of palladium" (2011). Axel Enders Publications. 27.

https://digitalcommons.unl.edu/physicsenders/27

This Article is brought to you for free and open access by the Research Papers in Physics and Astronomy at DigitalCommons@University of Nebraska - Lincoln. It has been accepted for inclusion in Axel Enders Publications by an authorized administrator of DigitalCommons@University of Nebraska - Lincoln. 


\title{
Anisotropy of zigzag chains of palladium
}

\author{
P. K. Sahota, ${ }^{1,2, a)}$ R. Skomski, ${ }^{2}$ A. Enders, ${ }^{2}$ D. J. Sellmyer, ${ }^{2}$ and A. Kashyap ${ }^{1,2}$ \\ ${ }^{1}$ The LNM Institute of Information Technology Jaipur, Rajasthan, India \\ ${ }^{2}$ Department of Physics and Astronomy and Nebraska Center for Materials and Nanoscience, \\ University of Nebraska, Lincoln, Nebraska 68588, USA
}

(Presented 18 November 2010; received 24 September 2010; accepted 30 November 2010; published online 1 April 2011)

\begin{abstract}
$A b$ initio calculations of the biaxial anisotropy of infinite Pd zigzag chains are presented. The simulations were performed with the Vienna Ab-Initio Simulation Package, using a tetragonal unit cell with a supercell approach where the atoms are repeated along the $z$-direction. The anisotropy is determined from the energies along the three principal directions [001], [100], and [010]. The second-order anisotropy constants $K_{1}$ and $K_{1}^{\prime}$ were extracted by fitting the calculated energy values to the phenomenological energy. The easiest magnetization direction is along the wire axis, whereas the hardest direction is perpendicular to the plane of the wire. The calculated anisotropy constants $K_{1}$ and $K_{1}^{\prime}$ are $5.5 \mathrm{MJ} / \mathrm{m}^{3}$ and $-13.9 \mathrm{MJ} / \mathrm{m}^{3}$, respectively These anisotropies are large, but they are smaller than those of linear chains, because the zigzag shape leads to a quenched orbital moment. @ 2011 American Institute of Physics. [doi:10.1063/1.3559505]
\end{abstract}

\section{INTRODUCTION}

Low-dimensional magnetism has long been a fascinating research area. Magnetism at low dimensions has fascinated researchers because of the drastically changed magnetic properties at these scales as compared to the bulk materials. One of the most important properties of magnetic materials is the magnetocrystalline anisotropy, which is exploited, for example, in permanent magnets and magnetic recording media. Low-dimensional nanostructuring offers unprecedented opportunities to tailor and enhance the magnetocrystalline anisotropy. ${ }^{1,2}$ Among the systems of current interest are chains of atoms that are enhanced to trend toward ferromagnetism due to band narrowing and the accompanying high density of states (DOS) at the Fermi level. ${ }^{3,4}$ Ironseries transition-metal nanowires with thicknesses of up to several nanometers exhibit interesting anisotropic properties, but our focus is on atomic nanowires. ${ }^{5}$

The magnetocrystalline anisotropies (MCAs) of various monatomic wires of the late transition metals $\mathrm{Fe}, \mathrm{Co}$, and $\mathrm{Ni}$ are high, ${ }^{6,7}$ but they are lower than those of some elemental $4 d$ and $5 d$ wires, where the spin-orbit coupling (SOC) is larger. Anisotropies as high as 6 to $20 \mathrm{meV} /$ atom have been calculated for free-standing linear monatomic Co wires. ${ }^{8}$ For $\mathrm{Ni}$ wires, the corresponding prediction is about $12 \mathrm{meV} /$ atom. ${ }^{9}$ These huge anisotropies are due to the rotational symmetry of the wires, which yields degenerate electron states and large, unquenched orbital moments. ${ }^{10}$ The corresponding anisotropy contributions are roughly linear in the spinorbit coupling constant $\lambda$, as compared to the second-order perturbation expression $\lambda^{2} / W$, where $W$ is the bandwidth. Even higher anisotropies have been predicted for linear monatomic Pt and Pd wires. These anisotropies are accompanied

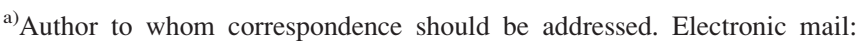
pankajsahota@gmail.com.
}

by a strong directional dependence of the magnetic moment termed "collossal." "3,11

Heavy transition metals such as Pd and Pt are nonmagnetic in their elemental forms but are easily spin-polarized in alloys and nanostructures due to their exchange-enhanced Pauli paramagnetism. In other words, the high density of states of Pd in bulk near the Fermi level suggests that a little push is sufficient to make to make the material ferromagnetic. In the calculations by Delin et al., ${ }^{12}$ the push was achieved by reducing the number of neighbors and increasing the surface area. For monatomic wires of Pd, this yields a magnetic moment of $0.7 \mu_{\mathrm{B}}$ per atom, and a magnetic anisotropy of nearly $5 \mathrm{meV}$ per Pd atom, or $75.5 \mathrm{MJ} / \mathrm{m}^{3}$, has been calculated by Blugel et al. ${ }^{11}$ However, due to crystalfield effects, ${ }^{10}$ any breaking of the rotational symmetry is expected to strongly reduce the anisotropy. This reduction is of great practical importance, because wires are typically embedded in a matrix or deposited on a substrate.

In the present paper, we calculate the second-order anisotropies of zigzag chains of Pd. Section II presents computational details, whereas Sec. III is devoted to the electronic structure and magnetic anisotropy. Finally, in Sec. IV, we discuss the physical meaning of the calculated anisotropy.

\section{COMPUTATIONAL DETAILS}

The calculations have been performed using the pseudopotential method as implemented in the Vienna Ab-Initio Simulation Package. ${ }^{13}$ The exchange and correlation effects are described by the method of Perdew and Wang, ${ }^{14}$ using a generalized gradient approximation within the framework of density-functional theory. Figure 1 shows the considered zigzag Pd chain, which lies in the $x-z$ plane and extends to infinity in the $z$-direction. To perform the calculations effectively, the chain is modeled by a standard supercell approach. A vacuum of five times the interatomic distance is added in both the $x$ - and $y$-directions, while the wire is infinite along 


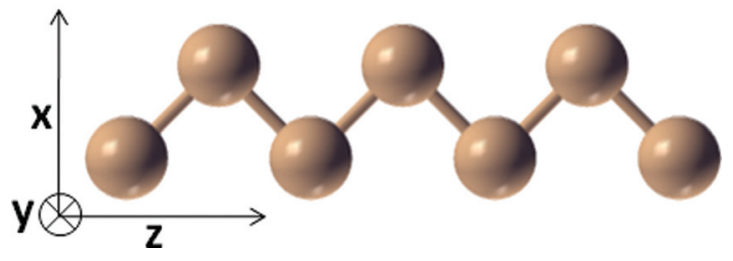

FIG. 1. (Color online) Schematic diagram for the zigzag chain of Pd.

the $z$-direction. The calculations for the nonspin-orbit part have been performed in an iterative self-consistent way, with $51 k$-points in the irreducible part of Brillouin zone and using the tetrahedron method. ${ }^{15}$

For the anisotropy calculations, $701 k$-points in the irreducible part of the Brillouin zone have been chosen. The spin-orbit interactions are included relativistically, using the second variation method. The anisotropy has been determined nonperturbatively from the spin-orbit coupling, that is, by diagonalization of the complete Hamiltonian. Fourth and higher-order relativistic corrections are not included, which is consistent with the second-order character of the anisotropy constants considered in this paper. The magnetic anisotropy energy of the system was obtained from the selfconsistently determined energies for the three different magnetization directions along the three principal axes of the system $(x, y$, and $z)$.

\section{ELECTRONIC STRUCTURE AND ANISOTROPY}

Both the magnetic moment and the anisotropy exhibit a strong dependence on the bond length. Figure 2 shows the total nonrelativististic energy as a function of the bond length. The energy optimization yields a stable minimum at a bond length of $3.48 \AA$, which corresponds to a maximum

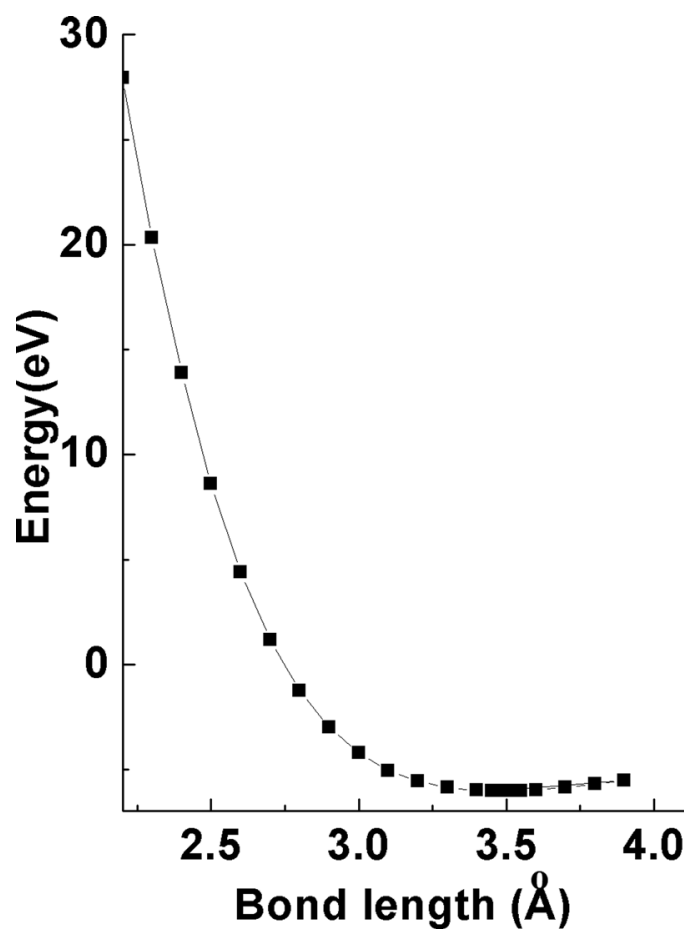

FIG. 2. Energy optimization curve for the zigzag chain of Pd. magnetic moment of $0.4405 \mu_{\mathrm{B}} /$ atom. The system under investigation here is a zigzag wire in a tetragonal supercell. The magnetocrystalline anisotropy energy for low-symmetry systems such as triclinic and orthorhombic crystals and the present zigzag wires is ${ }^{16}$

$$
\frac{E}{V_{\text {atom }}}=K_{1} \sin ^{2} \theta+K_{1}^{\prime} \sin ^{2} \theta \cos 2 \varphi .
$$

Here $K_{1}$ and $K_{1}^{\prime}$ are both first (or second) order anisotropy constants. $K_{1}^{\prime}$ can be ignored in uniaxial systems such as monatomic wires, ${ }^{17}$ but in the present case, the zigzag structure breaks the uniaxial symmetry and $K_{1}^{\prime}$ is generally comparable to $K_{1}$.

The calculations of total energy per unit cell for the system of zigzag Pd wires are performed for the three principal magnetization directions, namely, [001], [100], and [010]. The first-principle calculations yield $E_{001}=-6.223040 \mathrm{eV}$, $E_{100}=-6.223239 \mathrm{eV}$, and $E_{010}=-6.223476 \mathrm{eV}$. From Eq. (1) we derive the following relations between the anisotropy constants and the energies:

$$
\begin{gathered}
\frac{E_{001}}{V_{\text {atom }}}=K_{0} \quad(\theta=0, \phi=0), \\
\frac{E_{100}}{V_{\text {atom }}}=K_{0}+K_{1}+K_{1}^{\prime} \quad\left(\theta=90^{\circ}, \phi=0\right), \\
\frac{E_{010}}{V_{\text {atom }}}=K_{0}+K_{1}-K_{1}^{\prime} \quad\left(\theta=90^{\circ}, \phi=90^{\circ}\right) .
\end{gathered}
$$

Equations (2)-(4) yield

$$
\begin{gathered}
K_{0}=\frac{E_{001}}{V_{\text {atom }}}, \\
K_{1}=\frac{1}{2 V_{\text {atom }}}\left(E_{100}+E_{010}-2 E_{001}\right), \\
K_{1}^{\prime}=\frac{1}{2 V_{\text {atom }}}\left(E_{100}-E_{010}\right) .
\end{gathered}
$$

Using the calculated energies, we obtain $K_{1}=5.54 \mathrm{MJ} / \mathrm{m}^{3}$ and $K_{1}^{\prime}=-13.91 \mathrm{MJ} / \mathrm{m}^{3}$. These values are large and comparable to typical $L 1_{O}$ or rare-earth intermetallics, ${ }^{10}$ but they are about ten times smaller than the $K_{1}$ of a monatomic wire of Pd. ${ }^{11}$

\section{DISCUSSION AND CONCLUSIONS}

The signs of the two anisotropy constants mean that the easiest magnetization direction is along the $y$-direction, whereas the hardest axis is along the $z$-direction. The large negative value of $K_{1}^{\prime}$ shows that [001] is the harder direction, rather than the [010] direction.

In order to investigate why the anisotropy is smaller than that of monatomic Pd wires, we analyze the DOS for the system without SOC. Figure 3 shows the corresponding orbital-projected DOS. The main anisotropy contributions come from pairs of states that are located just above and below the Fermi level and are connected by spin-orbit matrix elements. The latter happens if the two orbitals have the same quantum number $m$ for the quantization axis parallel to 


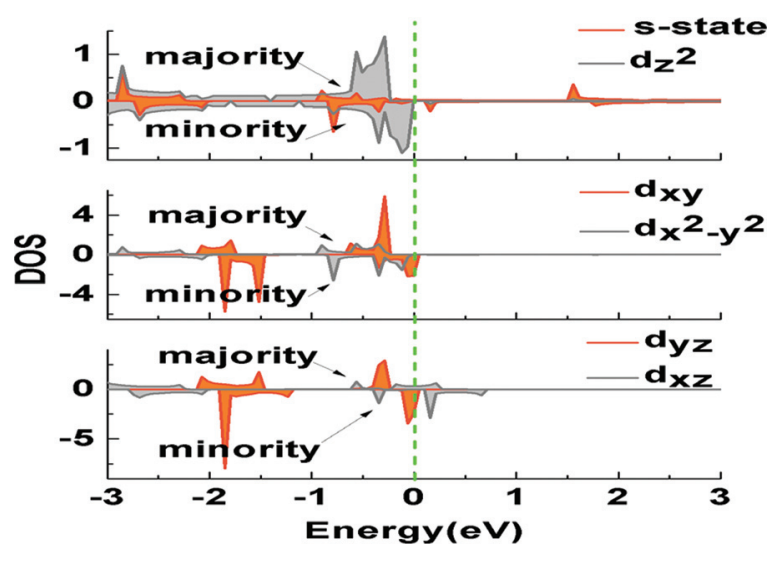

FIG. 3. (Color online) Density of states for the zigzag chain of Pd.

the magnetization. For example, the $d_{x y}$ and $d_{x}^{2}-y^{2}$ orbitals have $\left|m_{l}\right|=2$ and yield a strong easy-axis anisotropy contribution along the $z$-axis if they are located near the Fermi level. In linear wires, the rotational symmetry causes the $d_{x y}$ and $d_{x-y}^{2}{ }^{2}$ orbitals to be degenerate, which is the main reason for the high anisotropy of these wires.

In zigzag wires, the rotational symmetry is broken and the $d_{x y}$ and $d_{x-y}^{2}{ }^{2}$ states are no longer degenerate, as shown in Fig. 3. This effectively reduces the effect of the spin-orbit coupling. Physically, the large anisotropy of linear monatomic nanowires corresponds to the circular "orbital currents" around the wire axis, which yield a strong anisotropy contribution along the symmetry axis; in the zigzag case, though, there is a crystal field splitting energy due to the neighboring atoms, which reduces the anisotropy energy. ${ }^{10,16,17}$ Similar symmetry-breaking effects occur if a free-standing linear monatomic nanowire is deposited on a substrate or in a matrix.

The zigzag geometry of the present wire disrupts the circular orbital currents (quenching) and reduces the anisotropy. However, the relatively large SOC of $\mathrm{Pd}$ means the residual orbital moment and the anisotropy remain large.
In summary, we have investigated the second-order anisotropy constants for zigzag chains of $\mathrm{Pd}$. In addition to the anisotropy constant $K_{1}=5.54 \mathrm{MJ} / \mathrm{m}^{3}$, the low symmetry of the chain yields a nonuniaxial anisotropy contribution $K_{1}^{\prime}=-13.91 \mathrm{MJ} / \mathrm{m}^{3}$ to the MCA. The easiest magnetization direction is perpendicular to the wire plane, whereas the hard and intermediate axes are in the plane of the wire.

\section{ACKNOWLEDGMENTS}

The authors are thankful to the Department of Science and Technology, New Delhi through the Indo-European collaborative project "DYNAMAG", INT/EC/CMS(24/ 233552), and through Nano Mission, SR/NM/NS-20/2008. The work in Nebraska is supported by DOE (DJS), BREM (RS), ARPA-E (RS), ONR (RS), and NSF-MRSEC.

${ }^{1}$ J. Dorantes-Davila and G. M. Pastor, Phys. Rev. Lett. 81, 208 (1998).

${ }^{2}$ J. Hu, T. W. Odom, and C. M. Lieber, Acc. Chem. Res. 32, 435 (1999).

${ }^{3}$ A. Smogunov, A. D. Corso, A. Delin, R. Weht, and E. Tosatti, Nat. Nanotechnol. 3, 22 (2008).

${ }^{4}$ A. Bala, T. Nautiyal, and S. Auluck, J. Appl. Phys. 104, 014302 (2008).

${ }^{5}$ M. Paulus, F. Luis, M. Kröll, G. Schmid, and L. J. de Jongh, J. Magn. Magn. Mater. 224, 180 (2001).

${ }^{6}$ A. Stewart, J. Appl. Phys. 101, 09D503 (2007).

${ }^{7}$ O. N. Mryasov, V. A. Gubanov, and A. I. Liechtenstein, Phys. Rev. B 45, 12330 (1992).

${ }^{8}$ R. F. Medina, J. D. Davilla, and G. M. Pastor, New J. Phys. 4, 100.1 (2002).

${ }^{9}$ J. C. Tung and G. Y. Guo, Phys. Rev. B 76, 0944413 (2007).

${ }^{10}$ R. Skomski and J. M. D. Coey, Permanent Magnetism (Institute of Physics, Bristol, England, 1999).

${ }^{11}$ Y. Mokrousov, G. Bihlmayer, S. Heinze, and S. Blügel, Phys. Rev. Lett. 96, 147201 (2006).

${ }^{12}$ A. Delin, E. Tosatti, and R. Weht, Phys. Rev. Lett. 92, 057201 (2004).

${ }^{13}$ G. Kresse and J. Furthmüller, Phys. Rev. B 54, 11169 (1996).

${ }^{14}$ J. P. Perdew, J. A. Chevary, S. H. Vosko, K. A. Jackson, M. R. Pederson, D. J. Singh, and C. Fiolhais, Phys. Rev. B 46, 6671 (1990).

${ }^{15}$ P. E. Blöchl, O. Jepsen, and O. K. Anderson, Phys. Rev. B 49, 16223 (1994).

${ }^{16} \mathrm{R}$. Skomski, Simple Models of Magnetism (Oxford University Press, New York, 2008).

${ }^{17}$ M. Komelj, C. Ederer, J. W. Davenport, and M. Fähnle, Phys. Rev. B 66, 140407 (2002). 